\title{
Завершающая склеротерапия рецидивных и резидуальных варикозно расширенных вен нижних конечностей после хирургического и эндовенозных методов лечения
}

\author{
B. A. Мусаев \\ Медицинский факультет Университета Гази, г. Анкара, Турция
}

\section{Completing sclerotherapy of recurrent and residual varicosely changed veins of the lower extremities after surgical and endovenous methods of treatment}

\author{
V. A. Musayev \\ Medical Faculty of University of Gazi, Ankara, Turkey
}

\section{Реферат}

Цель. Улучшение результатов лечения пациентов с рецидивными и резидуальными варикозно расширенными венами и варикозными узлами нижних конечностей (НК), развившимися после хирургического и эндовенозного лазерного методов лечения.

Материалы и методы. У 32 больных (21 женщины и 11 мужчин) в возрасте от 28 до 71 года выполнили миниинвазивную завершающую склеротерапию рецидивных и резидуальных варикозно измененных вен и варикозных узлов, развившихся после хирургического и эндовенозного лазерного методов лечения в $45 \mathrm{HK}$.

Результаты. По данным контрольной цветной ультразвуковой допплерографии установлена полная окклюзия подкожных вен и варикозных узлов во всех 45 НК без их реканализации. Транзиторный тромбофлебит развился у 3 пациентов. На чувство инородного тела в виде мелких хорд жаловались 3 пациента, парестезию и ощущение онемения - 2 , экхимозы - 2. Эти осложнения не требовали специальных медикаментозных и физиотерапевтических методов лечения и спонтанно разрешились в течение 1 - 6 мес. Выраженных осложнений не наблюдали.

Выводы. У пациентов с неудовлетворительными результатами хирургического лечения хронической венозной недостаточности и варикозных узлов (частота рецидива составляет 48,1\%, резидуальной патологии подкожных вен - 25,0\%) требуется применение альтернативных миниинвазивных методов лечения. Комплексный подход и правильный выбор эндовенозных лазерных методов лечения позволяют достичь полной окклюзии подкожных вен у 97,37\% этих пациентов, сократить частоту возникновения рецидива заболевания и выраженных осложнений до минимума. Больным с реканализацией подкожных вен, а также развитием рецидива и резидуальных явлений в НК целесообразно выполнение завершающей склеротерапии под местной инфильтрационной анестезией в амбулаторных условиях или в стационаре.

Ключевые слова: хроническая венозная недостаточность; варикозные узлы; цветная ультразвуковая допплерография; стриппинг; эндовенозная лазерная абляция; радиофрекансная абляция; склеротерапия.

\section{Abstract}

Objective. Improvement of the treatment results in patients, suffering recurrent and residual varicosely changed veins and varicose nodes of the lower extremities, developed after surgical and endovenous laser methods of treatment.

Materials and methods. In 32 patients ( 21 women and 11 men) ageing from 28 to 71 yrs old, a miniinvasive definitive sclerotherapy of recurrent and residual varicosely changed veins and varicose nodes of the lower extremities, which have developed after surgical and endovenous laser methods of treatment in 45 patients, were performed.

Results. Complete occlusion of subcutaneous veins and varicose nodes of the lower extremities without their recanalization were revealed in 45 patients in accordance to data of the control color ultrasound dopplerography. Transitory thrombophlebitis have developed in 3 patients. There were following symptoms: a foreign body feeling like of a small chords - in 3 patients, paresthesia and feeling of numbness - in 2, and ecchimosis - in 2. These complications did not demand special medicinal and physiotherapeutic methods of treatment and spontaneously resolved during $1-6$ mo. Serious complications were absent. Complications. In patients with unsatisfactory results of surgical treatment of chronic venous insufficiency and varicose nodes (the recurrence rate constitutes $48.1 \%$, and in the residual pathology of subcutaneous veins - 25.0\%) the alternative miniinvasive methods of treatment must be applied. Complex approach and correct choice of endovenous laser methods of treatment permits to achieve a complete occlusion of subcutaneous veins in $97.37 \%$ of this patients, to reduce the rate of the disease recurrence and serious complications occurrence down to minimum. Conduction of a definitive sclerotherapy under local infiltrative anesthesia in ambulatory or stationary conditions is expedient in patients with the subcutaneous veins recanalization, development of recurrence and residual disease in the lower extremities.

Keywords: chronic venous insufficiency; varicose nodes; color ultrasound dopplerography; stripping; endovenous laser ablation; radio-frequency ablation; sclerotherapy.

Лечение хронической венозной недостаточности (XBH) в амбулаторных условиях с применением современных мелкодисперсных антикоагулянтов и компрес- сионных бандажей (чулок), которое уменьшает частоту развития тяжелых форм ХВН и намного снижает риск тромбоэмболии легочной артерии (ТЭЛА), возможно у 
60 - 70\% больных, но применяется менее чем у 1\% больных [1]. В настоящее время самыми щадящими, высокоэффективными и популярными методами считают эндовенозную лазерную и радиофрекансную абляцию с облитерацией большой (БПВ) и малой (МПВ) подкожных вен и их ветвей, а также перфорантных вен. Применение эндовенозной лазерной окклюзии эффективно у 80 - 97\% больных, сопровождается минимальными осложнениями, частота которых не превышает 3 - 10\% [2]. К сожалению, использование этих методик для лечения пациентов с ХВН ограничено, так как их выполняют на специальной дорогостоящей аппаратуре специально обученные высококвалифицированные специалисты-флебологи [3].

Цель исследования: улучшение результатов лечения больных с рецидивными и резидуальными варикозно расширенными венами и варикозными узлами НК, развившимися после хирургического и эндовенозного лазерного методов лечения.

\section{Материалы и методы исследования}

Нами в период с 2009 по 2015 г. на кафедре инвазивной радиологии медицинского факультета Университета Гази (г. Анкара, Турция) 32 больным после хирургического и эндовенозного лазерного методов лечения ХВН НК была выполнена миниинвазивная завершающая склеротерапия рецидивных и резидуальных варикозно измененных вен и варикозных узлов. Мужчин было 11, женщин 21 в возрасте от 28 до 71 года, средний возраст составил $(50,14 \pm 10,25)$ года (средняя величина $\pm S D)$.

Диагноз рецидивных и резидуальных варикозно измененных, в том числе расширенных, вен и варикозных узлов устанавливали и уточняли с помощью цветной ультразвуковой допплерографии (ЦУЗД). Данная методика позволяла выявлять локализацию и уровень варикозных узлов, реканализацию подкожных вен, наличие (отсутствие) в них рефлюкса венозной крови, а также проходимость глубоких вен. Использовали ультразвуковой аппарат LOGIQ 9 GE Ultrasound (Япония) сначала в вертикальном положении пациента, затем - в горизонтальном (на спине) и наоборот. В наружных подвздошных, поверхностной, глубокой и общей бедренной, задней большеберцовой артериях и задней артерии стопы был изучен и оценен тип кровотока с точки зрения острой (хронической) артериальной недостаточности.

Хирургическое лечение было проведено 40 больным на 52 НК. Кроссэктомия выполнена на 19 НК, флебэктомия на 11, стриппинг БПВ - на 9, МПВ - на 6, БПВ и МПВ - на 5, лигация БПВ - на 2. Большая доля паллиативных операций связана с тем, что у больных с высоким риском по мере возможности сохраняли БПВ для использования ее как наилучшего аутотрансплантата при выполнении дальнейших операций аорто-коронарного шунтирования.

Эндовенозная лазерная абляция (ЭВЛА) выполнена 126 больным на 164 НК: пораженной БПВ - на 131, МПВ - на 8, БПВ и МПВ - на 25. Радиофрекансная абляция (РФА) выполнена 16 больным на 26 НК: пораженной БПВ - на 16, МПВ - на 3, БПВ и МПВ - на 7.

Завершающую склеротерапию выполнили 32 больным на 45 НК: в системе МПВ - на 21, в системе БПВ - на 9, в системах МПВ и БПВ - на 15. Этиологически установлены рецидивный (на 29 НК) и резидуальный (на 16) варикоз, а также комбинированное варикозное поражение вен НК.

Методика ЭВЛА. При абляции в системе БПВ целесообразно супинационное, МПВ - пронационное положение пациента. Сначала фломастером отмечаем точку доступа по карте, указанной в руководстве по выполнению ЦУЗД. При недостаточности БПВ точка доступа находится в области коленного сустава или ниже его, где при допплерографии визуализируется первый расширенный участок вены, при недостаточности МПВ - в дистальной части НК. Затем под местной инфильтрационной (тумесцентной) анестезией (локальный анестетик, физиологический раствор или 8,4\% раствор натрия бикарбоната, раствор вазоконстриктора) в условиях контроля ЦУЗД лазерный файбер вводим в точку доступа. При недостаточности БПВ наконечник лазерного файбера располагаем на несколько сантиметров ниже сафено-феморального соустья, МПВ - сафено-поплитеального соустья [4].

Длина волны лазера варьирует от 810 до 1470 нм [5]. Мы применяли лазер с длиной волны 980 нм и силой 15 Вт. Под действием лазерной энергии происходят термальное повреждение интимы, денатурация коллагена в медии, в общем панмуральное повреждение венозной стенки вследствие внутрипросветного образования тромбов. Доза лазерной энергии которую выбирали в зависимости от диаметра пораженного сегмента вены, составляла от 60 до 100 Дж/см², так как после больших доз увеличиваются частота и тяжесть осложнений: экхимозов, парестезии, повреждения близлежащих подкожных и внутрикожных нервов, термических ожогов и асептического некроза кожи, тромбоза глубоких вен (ТГВ) и др. [6].

Процедуры РФА мы выполняли с использованием блока контроля радиофрекансной энергии ClosureRFG ${ }^{\mathrm{TM}}$ и системы с катетером ClosureFast ${ }^{\mathrm{TM}}$ (Covidien) по общепринятой методике. После обработки конечности антисептическим раствором и обертывания ее стерильным бельем в соответствующих точках доступа отдельно для БПВ и МПВ делали мелкие разрезы кожи скальпелем и под контролем ЦУЗД в месте разреза перкутанно пунктировали нужную подкожную вену иглой $18 \mathrm{G}$, через которую в вену вводили направляющий металлический стержень 0,035 inc. Затем иглу вытаскивали, над стержнем вводили интубационную систему, состоящую из 6F мембраны и 6F дилататора. Стержень вместе с дилататором извлекали, а через просвет 6F мембраны в вену вводили катетер ClosureFast $^{\mathrm{TM}}$. Под контролем ультразвукового исследования катетер продвигали в краниальном направлении - до 2 см соответствующего соединения. После этого по ходу пораженной подкожной вены в нескольких участках иглой $21 \mathrm{G}$ под кожу вводили смесь $0,9 \%$ физиологического раствора (1000 мл), 2\% раствора прилокаина (20 мл), $8,4 \%$ раствора натрия бикарбоната (20 мл) и 1 мг раствора адреналина. Под контролем ЦУЗД уточняли проходимость глубоких вен. Затем пациенту надевали чулок 2-го компрессионного класса и предлагали ходить в течение 30 - 40 мин. Спустя 3 ч пациентов выписывали.

методика склеротерапии. Некоторые авторы предпочитают склеротерапию использовать не изолированно, 
а в комбинации с другими методами. В последние годы в клинической практике больше применяют растворы полидоканола (Aethoxysclerol) и сульфата тетрадецила (Sotradecol) [7]. Если просвет вен и варикозных узлов не превышает 3 мм, можно вводить раствор препарата в виде жидкости, при большем просвете - в виде пены. Жидкостную и пенную склеротерапию используют также с целью облитерации рецидивных и резидуальных варикозных узлов вместе или после применения эндовенозных лазерных окклюзирующих технологий и выполнения хирургических вмешательств [8].

Для образования пены (foam) использовали технику Tessari: два шприца емкостью 10 мл соединяли между собой трехканальным наконечником [9], в один шприц набирали 1 - 2 мл склерозирующей жидкости, в другой - 4 - 8 мл воздуха и в течение 15 - 30 мин их беспрерывно смешивали до получения сгущенной пены, которую вводили прямо в просвет варикозных узлов, пораженных вен (ветвей), если их диаметр не превышал 3 мм. В вены больших калибров (диаметр более 3 мм) необходимо вводить пенный раствор под постоянным контролем ЦУЗД. Наличие рефлюкса в последнее время считают относительным показанием к выполнению пенной склеротерапии $[10,11]$.

Процедуру склеротерапии выполняли в позиции больного лежа в постели, начиная с тех участков, где варикозные узлы и вены (ветви) болыше поражены. До процедуры вены (варикозные узлы) должны быть опустошены поднятием конечности под углом более $60^{\circ}$ в течение несколько минут. В случае экстравазации препарата иглу немедленно вытаскивали, место пункции крепко прижимали пальцем на несколько минут, затем массажировали. При возникновении боли подкожно вводили 0,25\% раствор новокаина (3 - 5 мл) с целью анестезии и разбавления. После наполнения варикозных узлов, пораженных вен и их ветвей сразу прекращали введение пенного раствора для предотвращения его попадания в глубокую систему. Иглу извлекали, надевали чулок 2-го компрессионного класса на НК. Через 30 - 45 мин пациента выписывали.

Статистическую обработку проводили, применяя статистический пакет SPSS с версией 20.0. Для анализа использовали методы непараметрической статистики: критерий Манна-Уитни U-test - для независимых и Вилкоксона для зависимых переменных. Клинические симптомы и их интенсивность оценивали с помощью двух шкал: VCSS (Venous Clinical Severity Score - клиническая шкала оценки тяжести венозных заболеваний) и VAS (Visual Analog Scale - визуальная аналоговая шкала).

\section{Результаты}

До склеротерапии клинические симптомы и их интенсивность были оценены по VCSS в среднем в 3,1 бала - через 3 мес после хирургического лечения и в 1,4 бала - через 3 мес после эндовенозных процедур, после завершающей склеротерапии - в 2,8 и 1,3 бала соответственно $(\mathrm{p}<0,05)$.

До склеротерапии степень болевого синдрома по VAS была оценена в среднем в $(4,28 \pm 2,15)$ бала - через 3 мес после хирургического лечения и в $(2,86 \pm 1,94)$ бала - через 3 мес после эндовенозного лечения. Через месяц после завершающей склеротерапии эти показатели составили $(4,12 \pm 3,06)$ и $(2,77 \pm 1,24)$ бала соответственно $(\mathrm{p}<0,05)$.

Длина пораженных венозных ветвей составляла от 0,7 до 9 см, в среднем $(6,0 \pm 2,05)$ см (среднее значение $\pm \mathrm{SD})$, диаметр варикозных узлов - в среднем 5,6 мм (от 3 до 7,5 мм).

Склеротерапия была выполнена без технических сложностей и погрешностей.

После склеротерапии возникли в основном легкие осложнения: транзиторный тромбофлебит (3 наблюдения), чувство инородного тела в виде мелких хорд (3), парестезия и чувство онемения (2), экхимозы (2), которые не требовали соответствующих медикаментозных и физиотерапевтических методов лечения и спонтанно разрешились в течение 1 - 6 мес. ТГВ, ТЭЛА, ожогов и некроза кожи, подкожной клетчатки не наблюдали.

В общем во всех 45 конечностях с помощью ЦУЗД установлена полная окклюзия пораженных, патологически измененных сегментов вен и их ветвей, особенно варикозных узлов. При последующих контрольных осмотрах через 3, 6 и 12 мес клиническое выздоровление отмечено у всех больных без эстетических жалоб и дискомфорта.

\section{Обсуждение}

Долгие годы в лечении ХВН и связанных с ней варикозных узлов применяли травматичные объемные методы хирургических операций и химической эмболизации. По данным S. Dwerryhouse и соавторов после стриппинга и лигации БПВ частота возникновения рецидива рефлюкса и варикозных узлов составляла соответственно 29 и 71\% [12], по данным S. Sarin и соавторов - 18 и 45\%, а спустя 5 и 20 лет после операции у 20 - 80\% пациентов отмечена реккуренция заболевания [13]. По данным нашего исследования частота рецидива составила $48,1 \%$, резидуальной патологии подкожных вен - 25,0\%.

Первое исследование, посвященное результатам эндовенозных методов лечения ХВН и варикозных узлов опубликовано L. Navarro и R. J. Min в 2001 г.: ЭВЛА была выполнена 33 пациентам (40 конечностей) [14]. Через 4 мес полная окклюзия отмечена у всех больных. R. R. Van den Bos и соавторы в мета-анализе, охватывающем 119 научных исследований результатов лечения эндовенозными методами 12320 конечностей, выявили, что после стриппинга успешные результаты наблюдали у 78\%, химической склеротерапии - у 77\%, РФА - у 84\%, ЭВЛА - у 94\% пациентов [15]. N. Theivacumar и соавторы из 582 больных в возрасте 16 - 82 лет (644 НК) через 3 мес после ЭВЛА у 541 (93\%) больного (599 НК) констатировали полную окклюзию БПВ [6]. По данным контрольных исследований через три года после ЭВЛА и РФА G. B. Agus и соавторы из 1050 пациентов (1076 НК) у 1018 (97\%) отметили полную окклюзию пораженных поверхностных вен. Серьезных осложнений не наблюдали [2].

В нашем исследовании 142 пациентам (190 НК) были выполнены ЭВЛА и РФА. Полная окклюзия поверхностных подкожных вен отмечена на 185 (97,37\%), реканализация БПВ - только на 5 (2,63\%) НК. Преходящий тромбофлебит (8 наблюдений), парестезия (6) и экхимозы (3), возникшие после данных процедур, не требовали специаль- 
ных медикаментозных и физиотерапевтических методов лечения. Серьезных осложнений не было.

В нашем исследовании склеротерапия рецидивных и резидуальных поражений в системе подкожных вен после хирургических вмешательств и лазерных методов эндовенозной облитерации оказалась завершающей, после ее выполнения наблюдали полную окклюзию пораженных сегментов вен и их ветвей, в том числе варикозных узлов НК.

\section{Выводы}

1. В связи с неудовлетворительными результатами хирургического лечения пациентов с ХВН и варикозными узлами (частота рецидива - 48,1\%, резидуальной патологии подкожных вен - 25,0\%) требуется применение альтернативных миниинвазивных методов лечения. Комплексный подход и правильный выбор эндовенозных лазерных методов лечения этих пациентов позволяют увеличить частоту полной окклюзии подкожных вен до 97,37\%, сократить частоту возникновения рецидива заболевания и серьезных осложнений до минимума.

2. Больным с реканализацией подкожных вен, а также развитием рецидива и резидуальных явлений в НК целесообразно выполнение завершающей склеротерапии под местной инфильтрационной анестезией в амбулаторных условиях или в стационаре, после которой отмечены эстетичность, краткие сроки медико-социальной реабилитации, удовлетворенность пациентов и улучшение качества их жизни.

\section{References}

1. Fokin AA, Borsuk DA. Vozmojnosti endovenoznoy lazernoy obliteracii krupnyx pritokov bolshoy podkojnoy veny na bedre. Flebologiya (Russian). 2018;12(1):4-11 doi:10.17116/flebo20181214-11.

2. Agus GB, Mancini S, Magi G. The first 1000 cases of Italian Endovenouslaser Working Group (IEWG). Rationale, and long-term outcomes for the 1999-2003 period. Int Angiol. 2006 Jun;25(2).209-5. PMID:16763541.

3. Myers KA, Fris R, Jolley D. Treatment of varicouse veins by endovenouse laser therapy: assessment of results by ultrasound surveillance. Med J Aust. 2006 Aug 21;185(4).199-202. PMID:16922664 https:// www.mja.com.au/system/files/issues/185_04_210806/mye10280_fm.pdf.

4. Ozkan U. Endovenous Laser Ablation of Incompetent Perforator Veins: A New Technique in Treatment of Chronic Venaous Disease. Cardiovascular andispinterventional Radiology 2009;32:1067. PMID:19609603; doi:10.1007/s00270-009-9646-z.

5. Proebstle TM, Sandhofer M, Kargl A, Gül D, Rother W, Knop J, et al. Thermal damage of the iner vein wall during endovenous treatment: key role of energy absorption by intravascular blood. Dermatol Surg. 2002;28(7):596. doi:10.1046/j.1524-4725.2002.01309.x

6. Theivacumar N, Beale RJ, Mavor AI, Gough MJ. Factors influencing the effectiveness of Endovenous Laser Treatment (EVLT) for varicose veins due to saphenofemoral (SF) and long saphenous (LSV) reflux. Ireland TVSoGBa, editor. In The Vascular Society Yearbook London, UK; 2005:40. PMID:17936037. doi: 10.1016/j.ejvs. 2007.08.010

7. Goldman MP. Treatment of varicose and telangiectatic leg veins: doubleblind prospective comparative trial between aethoxysclerol and sotradecol. Dermatol Surg. 2002;28:52-5. PMID:11991271.

8. King T, Coulomb G, Goldman A, Sheen V, McWilliams S, Guptan RC. Experience with concomitant ultrasound-guidedisepifoam sclerotherapy and endovenous laser treatment in chronic venous disorder and its influence on Healt Related Quality of Life: interim analysis of more than 1000 consecutive procedures. Int Angiol. 2009;28:289-7. PMID: 19648872.

9. Tessari L, Cavezzi A, Frullini A. Preliminary Experience with a New Sclerosing Foam in the Treatment of Varicose Veins. Dermatol Surg. 2001;27:58. technique.PMID: 11231246. doi:10.1111/j.15244725.2001.00192.x

10. Dunst KM, Huemer GM, Wayand W, Shamiyeh A. Diffuse phlegmonous phlebitis after endovenous laser treatment of the greater saphenous vein. J Vasc Surg. 2006;43:1056-8. doi:10.1016/j.jvs.2006.01.030.

11. Sichlau MJ, Ryu RK. Cutaneous thermal injury after endovenous laser ablation of the great saphenous vein. J Vasc Interv Radiol. 2004;15:8657. PMID:15297592. doi:10.1097/01.RVI.0000136968.64870.07

12. Dwerryhouse S, Davies B, Harradine K, Earnshaw JJ. Stripping the long saphenous vein reduces the rate of reoperation for recurrent varicose veins: five-year results of a randomized trial. J Vasc Surg. 1999;29:5892. PMID- 10194484.

13. Sarin S, Securr JH, Coleridge Smith PD. Assessment of stripping the long saphenous vein in the treatment of primary varicose veins. Br J surg. 1992;79:889-3. doi:10.1002/bjs.1800790911.

14. Navarro L, Min RJ. Endovenous laser: a new minimally invasive method of treatment for varicose veins-preliminary observations using an $810 \mathrm{~nm}$ diode laser. Dermat Surg. 2001;27:117-2.

15. Van den Bos RR, Arends L, Kockaert M, Neumann M, Nijsten T. Endovenous therapies of lower extremity varicosities: a meta-analysis. J Vasc Surg. 2009;49:230-9. PMID: 18692348 doi: 10.1016/j.jvs.2008.06.030. 